\title{
Association of Epilepsy Surgery With Changes in Imaging-Defined Brain Age
}

Christophe E. de Bézenac, PhD, Guleed Adan, MBBS, Bernd Weber, MD, PhD, and Simon S. Keller, PhD

Neurology ${ }^{\circledR}$ 2021;97:e554-e563. doi:10.1212/WNL.0000000000012289
Correspondence

Dr. de Bézenac

cedb@liverpool.ac.uk

\section{Abstract}

\section{Objective}

To determine whether surgery in patients with mesial temporal lobe epilepsy (mTLE) is associated with reduced brain-predicted age as a neural marker overall brain health, we compared brain-predicted and chronologic age difference (brain age gap estimation [BrainAGE]) in patients before and after surgery with healthy controls.

\section{Methods}

We acquired 3D T1-weighted MRI scans for 48 patients with mTLE before and after temporal lobe surgery to estimate brain age using a gaussian processes regression model. We examined BrainAGE before and after surgery controlling for brain volume change, comparing patients to 37 age- and sex-matched controls.

\section{Results}

Preoperatively, patients showed an increased BrainAGE of more than 7 years compared to controls. However, surgery was associated with a mean BrainAGE reduction of 5 years irrespective of whether or not surgery resulted in complete seizure freedom. We observed a lateralization effect as patients with left mTLE had BrainAGE values that more closely resembled control group values following surgery.

\section{Conclusions}

Our findings suggest that while morphologic brain alterations linked to accelerated aging have been observed in mTLE, surgery may be associated with changes that reverse such alterations in some patients. This work highlights the advantages of resective surgery on overall brain health in patients with refractory focal epilepsy.

From the Department of Pharmacology and Therapeutics (C.E.d.B., G.A., S.S.K.), Institute of Systems, Molecular and Integrative Biology, University of Liverpool; The Walton Centre NHS Foundation Trust (C.E.d.B., G.A., S.S.K.), Liverpool, UK; and Institute of Experimental Epileptology and Cognition Research (B.W.), University of Bonn, Germany.

Go to Neurology.org/N for full disclosures. Funding information and disclosures deemed relevant by the authors, if any, are provided at the end of the article. 


\section{Glossary}

BrainAGE = brain age gap estimation; $\mathbf{C I}=$ confidence interval; $\mathbf{H S}=$ hippocampal sclerosis; ILAE = International League Against Epilepsy; MAE = mean absolute error; $\mathbf{m T L E}=$ mesial temporal lobe epilepsy; PBVC = percentage brain volume change; PS = persistent seizures; $\mathbf{S F}=$ seizure/aura-free; T1W = T1-weighted.

Mesial temporal lobe epilepsy (mTLE) is one of the most common forms of focal epilepsy ${ }^{1}$ associated with a number of pathologic alterations linked to premature brain aging. ${ }^{2}$ For one-third of patients with mTLE, antiseizure medication is ineffective, ${ }^{3}$ leading to surgery program referrals that aim to localize and resect the epileptogenic zone., ${ }^{4,5}$ Between $27 \%$ and $67 \%$ of patients who have surgery become seizure-free. ${ }^{6}$

Despite a risk of cognitive deterioration related to residual function in resected brain tissue, surgery can result in neuropsychologic improvements, ${ }^{7}$ particularly when seizures are controlled and drug load reduced. ${ }^{8}$ The imaging literature provides evidence for postoperative brain network plasticity in support of restorative brain function. ${ }^{9,10}$ However, there is no reliable biomarker to assess the effect of surgery on overall brain health.

A machine learning model for estimating chronologic age from structural MRI scans has shown promise. ${ }^{11,12}$ Increased brain-predicted age (relative to actual age) indicates accelerated aging or higher cumulative exposure/sensitivity to pathologic brain insults, in contrast to brain resiliance. ${ }^{13}$ The brain age gap estimation (BrainAGE) has been used to examine neurodegenerative and psychiatric disorders and the influence of gene interaction, environment, and life burden. ${ }^{14}$ In epilepsy, increased BrainAGE was observed in patients with refractory focal epilepsy ${ }^{15}$ and patients with temporal lobe epilepsy (TLE) and interictal psychosis. ${ }^{16}$

In this study we tested the effect of neurosurgery on BrainAGE as a measure of overall brain health, comparing 48 patients with mTLE before and after surgery to 37 controls. We expected patients to have higher BrainAGE but that successful surgery would be associated with an overall decrease.

\section{Methods}

\section{Participants}

We analyzed structural T1-weighted (T1W) MRI obtained from 48 patients ( 25 female) with refractory mTLE and neuroradiologically defined unilateral hippocampal sclerosis (HS) (mean age 39.08 years, SD 12.73) who underwent amygdalohippocampectomy at University Hospital Bonn, Germany. Patients ( $n=48)$ were part of a consecutive series of patients ( $n$ $=115$ ) being considered for surgery who enrolled in the study and had both pre- and postoperative T1W data suitable for analysis. ${ }^{18}$ After confident diagnosis of unilateral mTLE based on standard clinical protocols and detailed presurgical evaluation, patients underwent selective amygdalohippocampectomy ${ }^{17}$ in either the left $(n=17)$ or right $(n=31)$ hemisphere, with subtemporal $(\mathrm{n}=21)$ or transsylvian $(\mathrm{n}=27)$ access. ${ }^{18}$ Presurgical evaluation included interictal EEG with video monitoring and, where clinically required, additional intracranial electrode recording, MRI scanning, and neuropsychologic testing. HS diagnosis was made by a neuroradiologist experienced in epilepsy lesion detection on the basis of hippocampal volume loss and structural alterations observed on the MRI scans. ${ }^{19} \mathrm{HS}$ was histologically confirmed in all resected specimens. Follow-up structural T1W scans were acquired for all patients after surgery (mean 1.56 years, SD 0.99, between surgery and the follow-up scan; mean 1.96 years, SD 0.92, between first and second scans). The International League Against Epilepsy (ILAE) outcome classification system was used for postoperative seizure outcome follow-up $^{20}: 26$ were rendered seizure/aura free (SF) (ILAE I) while 22 patients continued to have seizures (persistent seizures [PS]) (ILAE II-VI) following surgery. Clinical data for patient outcome groups is outlined and compared in the table. We also analyzed MRI from a sample of 37 neurotypical controls similar in age and sex to the individuals who underwent surgery for epilepsy (mean age 40.08 years, SD 13.94; 21 female).

\section{Imaging}

All participants were scanned on a 3T scanner (Magnetom Trio, Siemens) and an 8-channel head coil at the Life \& Brain Center in Bonn. Three-dimensional T1W magnetizationprepared rapid gradient echo images were used for the BrainAGE analysis (160 slices, repetition time $51,300 \mathrm{~ms}$, inversion time 5,650 ms, echo time $53.97 \mathrm{~ms}$, resolution $1.0 \times$ $1.0 \times 1.0 \mathrm{~mm}^{3}$, flip angle $10^{\circ}$ ).

\section{Brain Age Prediction}

Brain-predicted age was computed from raw T1W MRI scans using the BrainAgeR analysis pipeline (github.com/jamescole/brainageR), previously described in detail. ${ }^{12}$ The pipeline includes the segmentation and normalization of MRI with SPM12's DARTEL toolbox. ${ }^{21}$ The quality of tissue segmentation was systematically assessed for all participants and no errors were found through visual inspection of segmentation output. After CSF segmentation masking, preprocessed gray and white matter images were vectorized and concatenated. These data were then entered into a principal components analysis to reduce dimensionality. Components for the top $80 \%$ of variance were used $(n=435)$ for brain age prediction in a machine-learning algorithm based on a pretrained Gaussian process regression model implemented in R package Kernlab. ${ }^{22}$ This model was trained on scans of 3,377 healthy individuals from 7 publicly available datasets ${ }^{12}$ and tested on 611 different scans of healthy individuals aged between 18 and 90 years. The model accurately predicted chronologic age $(r=$ $0.95, R^{2}=67.24 \%$, MAE 4.9 years). As with other brain age 
Table Clinical Demographics

\begin{tabular}{|c|c|c|c|c|}
\hline & $P S(n=22)$ & $S F(n=26)$ & Total $(n=48)$ & $p$ Value \\
\hline \multicolumn{5}{|l|}{ Age at scan 1,y } \\
\hline Mean (SD) & 41.318 (14.397) & $37.192(11.064)$ & 39.083 (12.729) & \\
\hline Range & $16.000-70.000$ & $19.000-59.000$ & $16.000-70.000$ & \\
\hline \multicolumn{5}{|l|}{ Age at scan 2, y } \\
\hline Mean (SD) & $43.273(14.190)$ & 39.154 (10.997) & $41.042(12.593)$ & \\
\hline Range & $19.000-72.000$ & $20.000-61.000$ & $19.000-72.000$ & \\
\hline Age at onset, y & & & & 0.997 \\
\hline Mean (SD) & $15.682(12.171)$ & $15.692(10.646)$ & $15.688(11.246)$ & \\
\hline Range & $1.000-42.000$ & $1.000-35.000$ & $1.000-42.000$ & \\
\hline Age at surgery, $y$ & & & & 0.267 \\
\hline Mean (SD) & 41.727 (14.548) & 37.577 (11.017) & 39.479 (12.786) & \\
\hline Range & $17.000-72.000$ & $19.000-59.000$ & $17.000-72.000$ & \\
\hline Sex, n (\%) & & & & 0.049 \\
\hline $\mathbf{F}$ & $15(68.2)$ & $10(38.5)$ & $25(52.1)$ & \\
\hline M & $7(31.8)$ & $16(61.5)$ & $23(47.9)$ & \\
\hline Surgery side, n (\%) & & & & 0.368 \\
\hline Left & $16(72.7)$ & 15 (57.7) & $31(64.6)$ & \\
\hline Right & $6(27.3)$ & $11(42.3)$ & $17(35.4)$ & \\
\hline Surgery access, n (\%) & & & & 0.561 \\
\hline Subtemporal & $11(50.0)$ & $10(38.5)$ & $21(43.8)$ & \\
\hline Transsylvian & $11(50.0)$ & $16(61.5)$ & $27(56.2)$ & \\
\hline
\end{tabular}

Clinical demographics of 48 patients with mesial temporal lobe epilepsy who underwent surgery. Patients are divided between those who became seizurefree (SF) and those who had persistent seizures (PS) following surgery. $p$ Values were determined by a Fisher exact test for categorical variables and an analysis of variance test for continuous variables.

models, a proportional bias was observed where chronologic age correlated with the difference between brain predicted and actual age $(r=-0.379){ }^{23}$

\section{Brain Change Control}

To control for between-patient differences in overall brain volume change following surgery we used SIENA, part of FSL. $^{24}$ Brain and skull images were first extracted from the 2 -timepoint whole-head input data. The 2 brain images were then aligned to each other and resampled into a space halfway between the two. Tissue segmentation was then performed to identify nonbrain/brain edge points. ${ }^{25}$ The perpendicular displacement of edges between the 2 timepoints was then estimated at these edge points. Using mean edge displacement, a whole brain estimate of percentage brain volume change (PBVC) between the 2 timepoints was computed.

To ensure that the surgical cavity did not significantly bias the BrainAGE measurement, we also implemented an automated lesion-filling procedure ${ }^{26}$ previously tested in relation to BrainAGE in a large $(\mathrm{n}>500)$ multiple sclerosis cohort. $^{27}$

\section{Statistical Analysis}

Statistical analyses were performed in R (v 3.6.0). Fisher exact test was used for categorical variables and an analysis of variance test for continuous variables to compare demographic differences between clinical outcome groups (table). All were nonsignificant $(p>0.05)$, with the exception of sex, which was included as a control variable along with age in subsequent statistical modeling.

BrainAGE was calculated as the brain-predicted age minus chronologic age at the time of the MRI scan. As there was no evidence of non-normal distribution $(\mathrm{W}=0.98, p=0.150)$ and inhomogeneity of variance between groups (FlingerKilleen: med $\left.\chi^{2}[2]-1.43 ; p=0.489\right)$ in the data, we used a linear multiple regression model to compare BrainAGE 


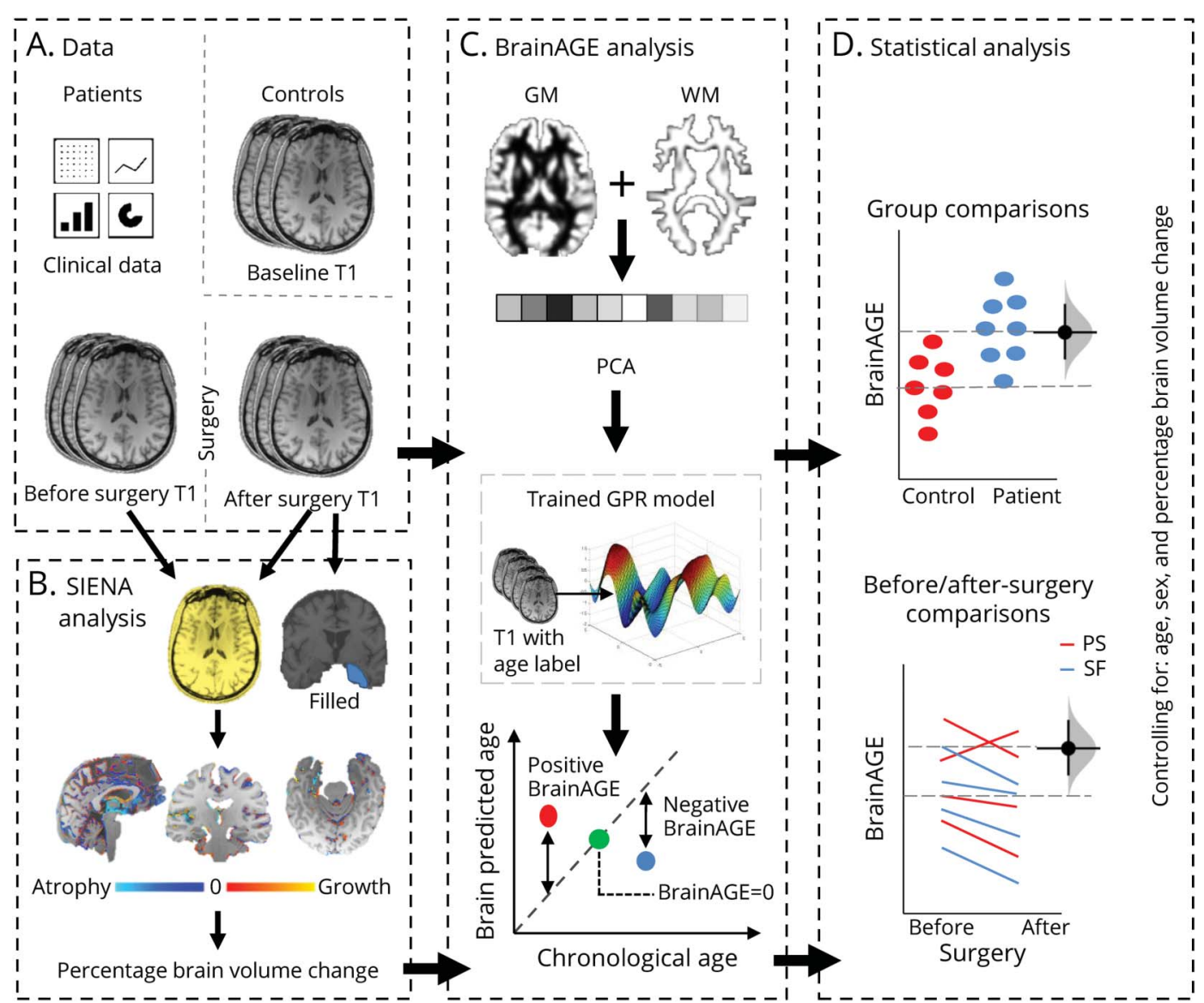

(A) In addition to clinical patient data, 3D T1-weighted MRI scans were acquired for 48 patients with mesial temporal lobe epilepsy before and after temporal lobe surgery and for 37 controls. (C) Brain age was estimated for each scan using a trained gaussian processes regression (GPR) model following tissue segmentation, vectorization, and principal components analysis (PCA)-based dimension reduction. Brain age gap estimation (BrainAGE) was computed as brain-predicted age minus actual age. BrainAGE comparisons were made between patients and control groups before and after epilepsy surgery, controlling for percentage brain volume change (SIENA; B), age, and sex (D). GM = gray matter; WM = white matter.

between controls and patient groups (SF, PS, left mTLE, right mTLE) both before and after surgery. Age was included as a covariate in all models to correct for the previously reported proportional bias, ${ }^{28}$ in addition to sex, gray matter, white matter, and CSF brain volume. Figure 1 is a graphic representation of the study analysis pipeline. To directly compare BrainAGE before and after surgery within patient participants and across patient groups, we used a repeated-measures mixed model with patients' ID included as a random effect and PBVC, resection size, age, sex, and brain tissue volumes as control variables.

\section{Standard Protocol Approvals, Registrations, and Patient Consents}

All patient and control participants provided written informed consent and the local ethics committee approved this study.

\section{Data Availability}

The data that support the findings of this study are available via the corresponding author, on reasonable request.

\section{Results}

In accordance with the findings of previous work, ${ }^{27}$ we found that the difference between BrainAGE for unfilled and filled postoperative scans was also not significant in our data (mean difference $-0.79,95 \%$ confidence interval $[\mathrm{CI}]-5.66$ to $4.09, t$ [37] $-0.33, p=0.745)$, suggesting that the pipeline is robust to surgical cavity-related bias. Figure 2 shows T1W images (first row) before and after surgery for 2 patients, one with right and the other with left-lateralized medial mTLE. The associated mask (CSF) and gray and white matter segmentations used in the computation of BrainAGE are also included for original (unfilled) and filled images. As the figure indicates, resections were appropriately masked out (see row 2 ) of gray and white matter segmentations in original images for all patients. Resections in filled images tended to be included in white matter segmentations. We therefore used BrainAGE computed from original images for the analysis.

Brain predicted age was highly correlated with chronologic age $(r=0.91,95 \%$ CI $0.83-0.95)$ in controls with a mean 
Figure 2 T1-Weighted Images Before and After Surgery for 2 Patients, 1 With Right- and the Other With Left-Lateralized Mesial Temporal Lobe Epilepsy (mTLE)

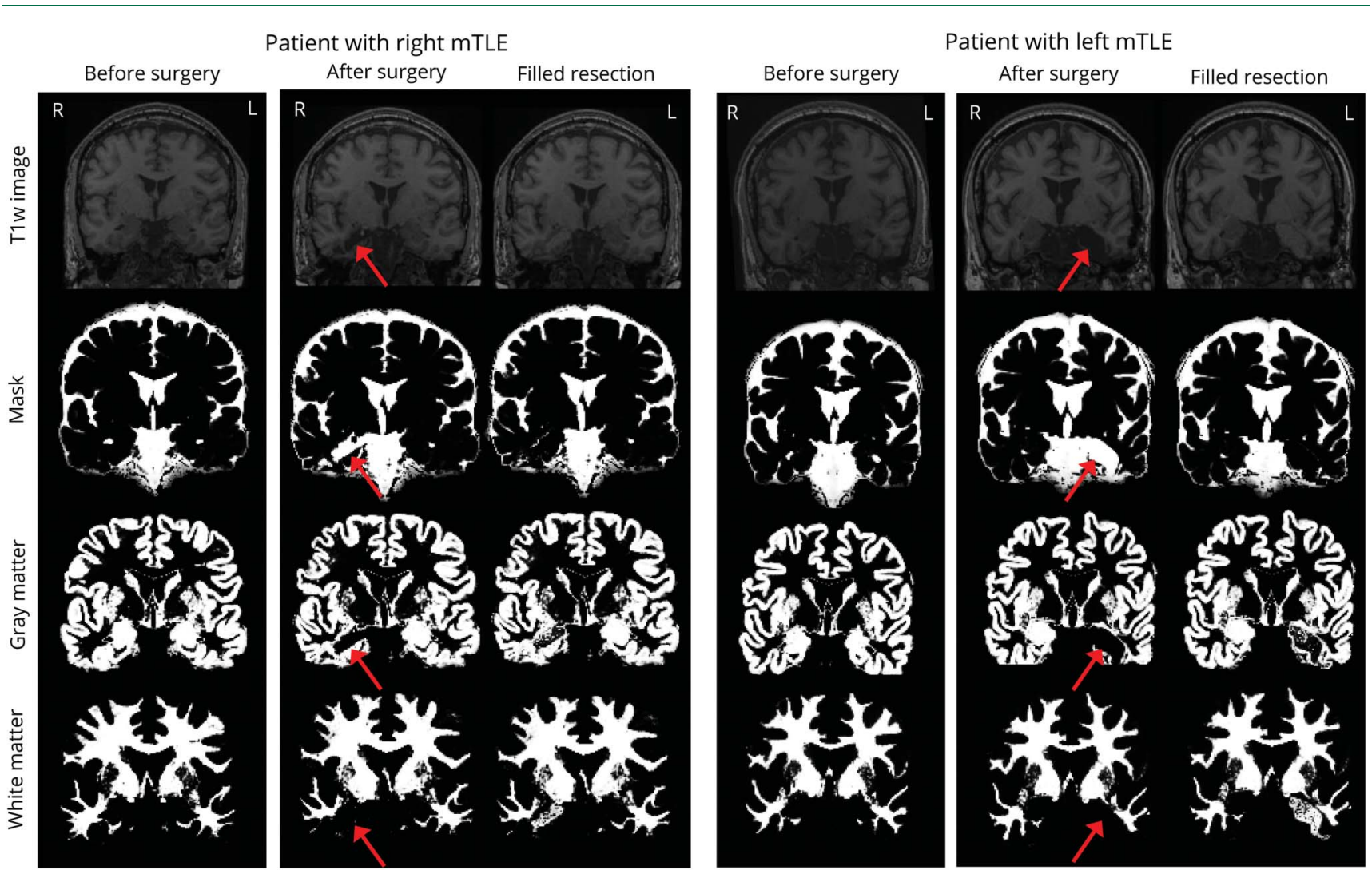

Below the associated masks, CSF and gray and white matter segmentations used in the computation of brain age gap estimation (BrainAGE) are included for original (unfilled) and filled images following surgery. Resection location is indicated by a red arrow for original images used in the analysis.

absolute error of 4.08 years, comparable to the MAE found in the original training datasets (MAE 4.9) ${ }^{12}$ The mean $( \pm$ SD) BrainAGE in controls was $-0.68( \pm 5.85)$ years. There was a significant correlation between BrainAGE and chronologic age in controls ( $r=-0.45,95 \% \mathrm{CI}-0.67$ to -0.14$)$, in line with the previously reported proportional bias. ${ }^{28}$

Figure 3 shows group differences with estimation graphics implemented in dabestr. ${ }^{29}$ Data points are displayed as a swarmplot with effect size presented on an aligned axes as a $95 \%$ CI calculated through bootstrap sampling $(n=5,000)$. On average, patients with epilepsy showed an increased BrainAGE of 7.97 years compared to controls prior to surgery (95\% CI $5.26-10.8$ ) and an increase of 2.8 years following surgery ( $95 \%$ CI 0.05-5.78). BrainAGE differences between controls and patient subgroups before surgery were as follows: SF 8.71 (95\% CI 5.65-12.3); PS 7.09 (95\% CI 3.69-10.6); left mTLE 6.73 (95\% CI 3.58-9.66); right mTLE 10.2 (95\% CI 7.03-14.8). BrainAGE differences between controls and patient subgroups after surgery were as follows: SF 3.37 (95\% CI 0.161-7.07); PS 2.13 (95\% CI -1.31 to 5.86); left mTLE 0.16 (95\% CI -2.78 to 3.17); right mTLE 7.61 (95\% CI 4.45-11.7). Plots in figure 3 show increased BrainAGE in patient groups (PWE, SF, PS, left, right) compared to controls before but not after surgery, with greatest postoperative reduction occurring for patients with left mTLE.

The linear regression model used to evaluate group differences in baseline BrainAGE (before surgery and correcting for age and sex, gray matter, white matter, and CSF brain volume) explained a significant and substantial proportion of variance $\left(R^{2}=0.60,90 \%\right.$ CI $\left.0.44-0.68, R_{a d j}^{2}=0.57\right)$. Significantly higher BrainAGE was found for both SF $(b=5.47$, 95\% CI $2.70-8.25)$ and $P S(b=4.69,95 \%$ CI $1.87-7.50]$ patient groups compared to controls. However, the estimation marginal means ${ }^{30}$ indicated that BrainAGE difference between SF and PS was not significant $(t[76]=0.52, p=0.862)$. Patient outcome groups were also not significantly different from controls using postoperative BrainAGE values (model fit $=R^{2}=0.50$, $90 \%$ CI 0.32-0.59, $R_{a d j}^{2}=0.46 ; \mathrm{SF}=\mathrm{b}=-0.07,95 \% \mathrm{CI}-2.90$ to 2.77 ; PS $=\mathrm{b}=-0.17,95 \% \mathrm{CI}-3.05$ to 2.71 ). Although resection size did not differ between patients with left- and right-lateralized $\operatorname{mTLE}(\mathrm{t}[44]=-0.03, p=0.976)$, grouping patients by lateralization again revealed higher BrainAGE before surgery for both groups compared to controls (left mTLE $=\mathrm{b}=3.72,95 \% \mathrm{CI} 1.23-6.21$; right $\mathrm{mTLE}=\mathrm{b}=7.57,95 \% \mathrm{CI}$ $4.62-10.52)$ and a significant left vs right difference $(\Delta M=-3.85,95 \%$ CI -7.39 to -0.31$)$. Following surgery, 


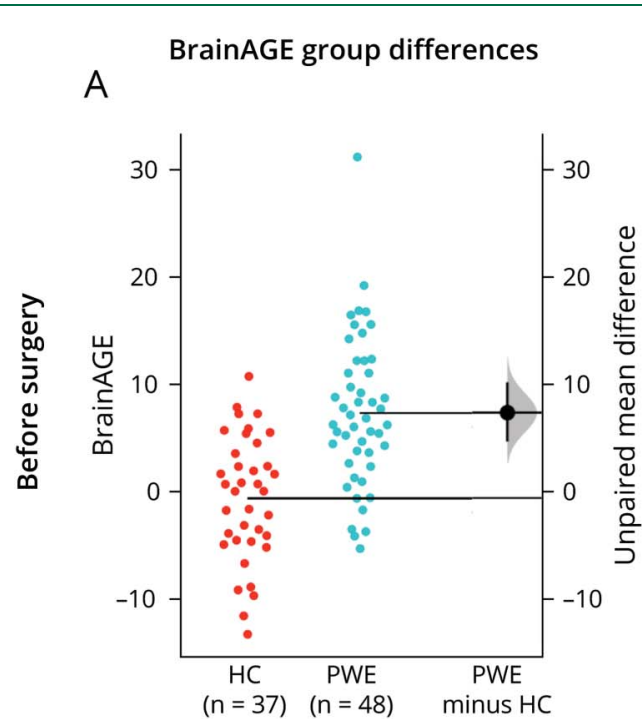

D

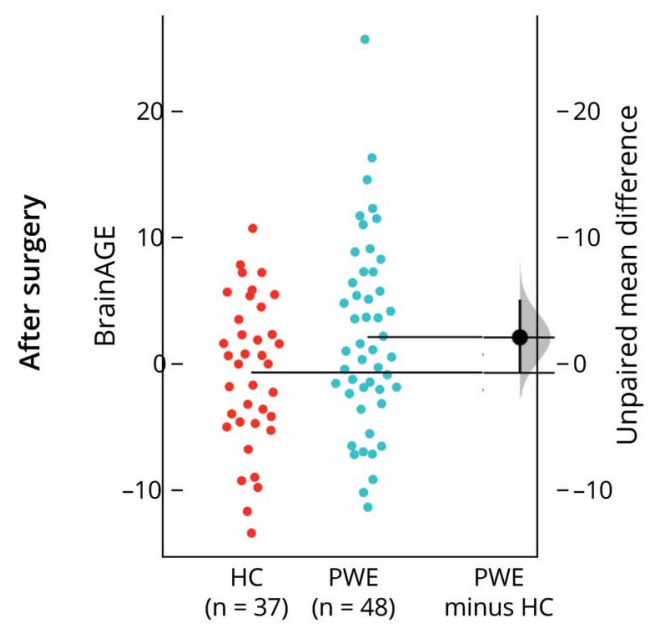

B
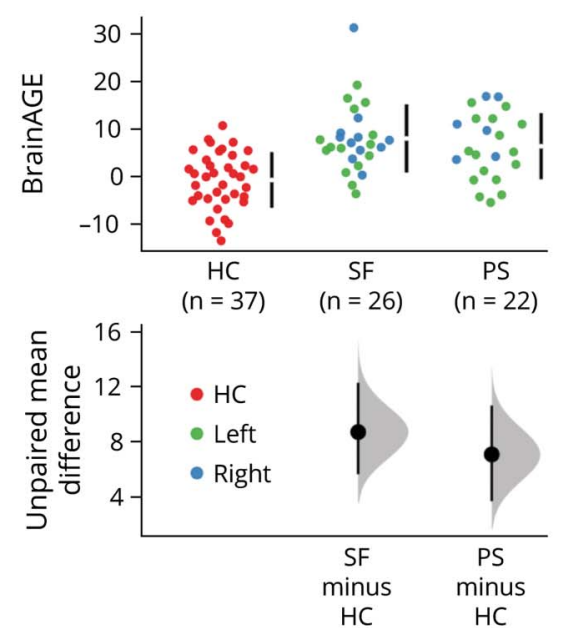

$\mathrm{E}$

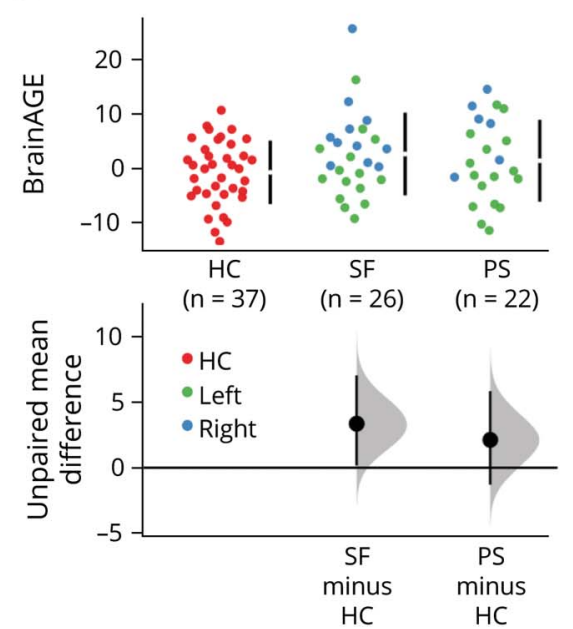

C

mTLE side

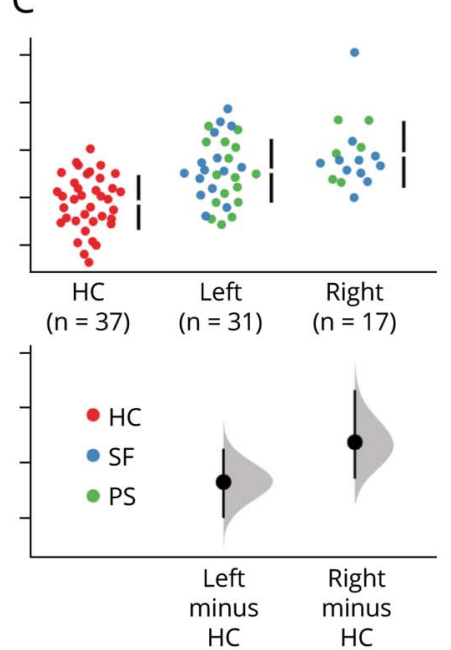

F

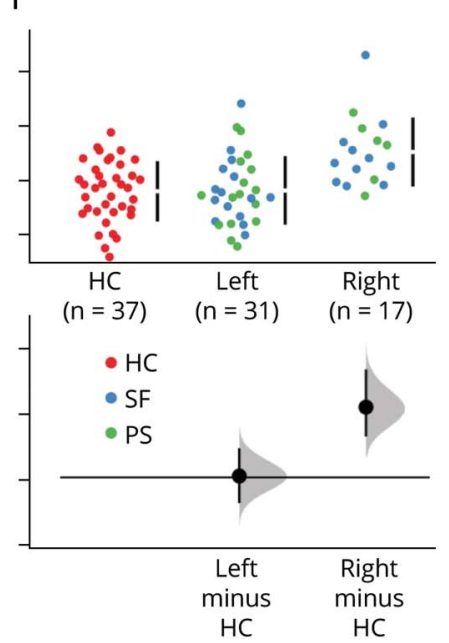

Comparisons of BrainAGE (computed as brain-predicted age minus actual age) for patients with epilepsy (PWE) and healthy controls (HC) (A, D), patients who are seizure-free (SF) and those with persistent seizures (PS) following surgery (B, E), and left- and right-sided mesial temporal lobe epilepsy (mTLE) (C, F). Comparisons are presented with BrainAGE values before $(A-C)$ and after (D-F) surgery. In $A$ and $D$, raw data points for BrainAGE in HC and PWE are shown in the left panel with unpaired group difference estimations plotted as a bootstrap sampling distribution $(n=5,000)$ (shaded area). Average effect size (mean difference) is depicted as a black dot and the $95 \%$ confidence interval ( $\mathrm{Cl}$ ) indicated by the ends of the vertical error bar. In B, C, E, and F, raw data for patient subgroups are compared to $\mathrm{HC}$ (red) and plotted/color coded on the upper panel with associated estimation plots shown below. Note that where the $95 \% \mathrm{Cls}$ (error bars) cross the horizontal line at zero, an effect size equal to zero is possible, i.e., no reliable difference from HC.

however, patients with right $(\mathrm{b}=7.46,95 \% \mathrm{CI} 3.83-11.09)$ but not left $(\mathrm{b}=0.25,95 \% \mathrm{CI}-2.72$ to 3.23$)$ mTLE had significantly higher BrainAGE compared to controls, with marginal means estimation showing right vs left difference to be more significant in postoperative BrainAGE $(\Delta M=-7.20,95 \% \mathrm{CI}$ -11.71 to -2.70$)$.

Figure 4 shows paired mean difference estimation plots of BrainAGE for different patient groups. The average difference after compared to before surgery was -5.17 years (95\% CI -6.53 to -3.91$)$. The total explanatory power of the repeatedmeasures mixed model used to assess the effect of surgery on BrainAGE was substantial (conditional $R^{2}=0.87$ ) and the part related to the fixed effects alone (marginal $R^{2}$ ) was 0.31 . As can be seen in the paired difference plot presented in figure
4, the main effect of surgery was large with BrainAGE significantly higher $(M=5.17$ years $)$ before compared to after surgery $(\beta 7.34$, SE 0.97 , standardized $\beta 0.94, p<0.001)$. The effect of outcome (SF-PS) was not significant $(\beta-0.70$, SE 1.67 , standardized $\beta-0.09, p=0.677)$, although a trend emerged when only patients with continuing seizures with loss of awareness (ILAE 3-6) were included into the PS group $(\beta-3.05$, SE 1.61, $p=0.065)$. The interaction effect of surgery and outcome was not significant (PS = ILAE $2-6: \beta-0.80$, SE 1.24, standardized $\beta-0.10, p=0.519$; PS $=$ ILAE 3-6: $\beta-0.39$, SE 1.35, standardized $\beta-0.05, p=0.773)$. The main effect of lateralization (right/left mTLE/surgery) was large and significant with an overall increased BrainAGE associated with right lateralization ( $\beta 8.05$, SE 1.58, standardized $\beta 1.05, p<$ 0.001 ). The interaction between surgery (BrainAGE before 


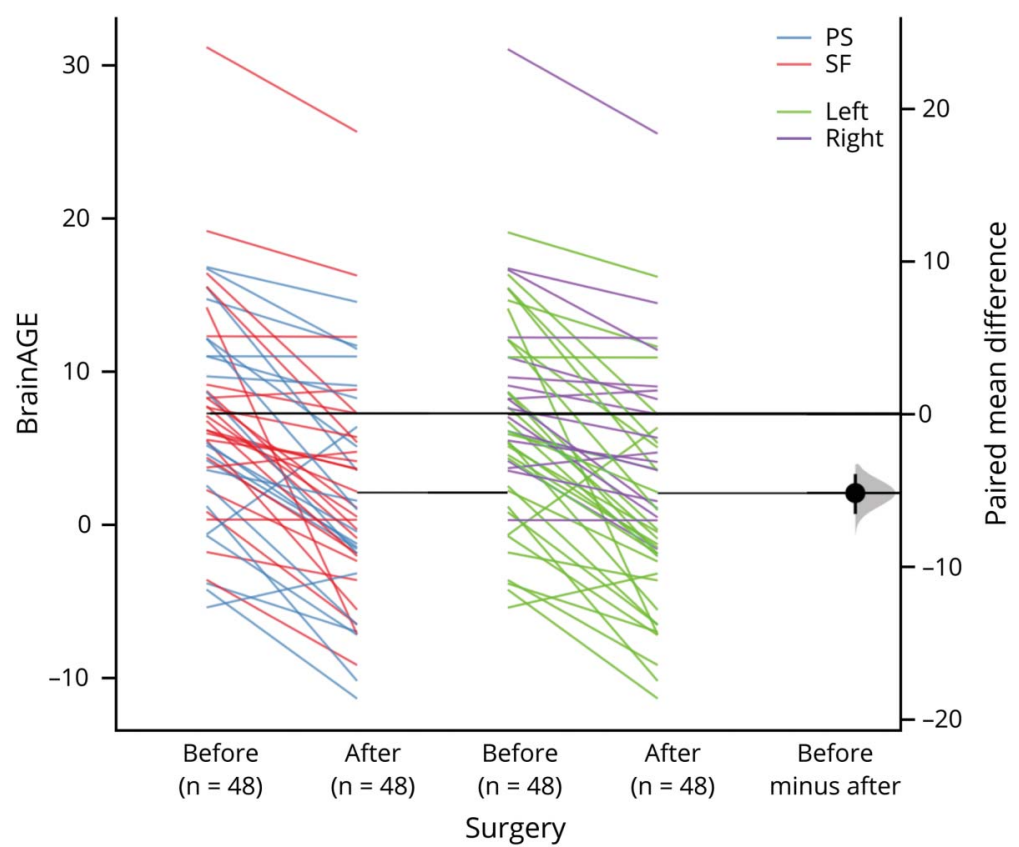

Paired difference of BrainAGE (computed as brain-predicted age minus actual age) before and after epilepsy surgery in patients with mesial temporal lobe epilepsy (mTLE). Colors indicate patient subgroups (PS = persistent seizures; $\mathrm{SF}=$ seizure-free; left = left mTLE; right = right mTLE). Paired differences are shown on the right with confidence plotted as a randomized bootstrap sampling distribution ( $n=5,000)$ (shaded area) and the average effect size depicted as a black dot and the $95 \%$ confidence interval indicated by the ends of the vertical error bar. and after) and lateralization was also significant, indicating significantly less postoperative BrainAGE reduction for right compared to left mTLE $(\beta-3.71$, SE 1.22, standardized $\beta$ $-0.48, p<0.01)$. The main effect of resection size on BrainAGE was not significant $(\beta 0.75$, SE 0.80 , standardized $\beta 0.10$, $p=0.347)$ and there was a small positive effect of PBVC $(\beta$ 1.00 , SE 0.48 , standardized $\beta 0.21, p<0.05$ ). Other variables such as age at seizure onset, years from onset to surgery, surgery access, and seizure frequency and burden were removed from the final model due to minimal and nonsignificant effects on BrainAGE.

\section{Discussion}

We used a brain-predicted age measure to investigate the effects of epilepsy surgery on overall brain health. Preoperatively, patients showed an increased BrainAGE (difference between brain predicted and actual age) of more than 7 years compared to controls. However, surgery was associated with a BrainAGE reduction of an average of 5 years irrespective of whether the procedure resulted in seizure freedom. This postoperative reduction was particularly pronounced for patients with left lateralized mTLE, where BrainAGE values following surgery resembled those of controls.

Given the correlation between BrainAGE and cognitive decline, ${ }^{31}$ the postoperative normalization of BrainAGE is consistent with literature that shows restoration of some aspects of neuropsychologic function following successful epilepsy surgery. This has been observed in domains including verbal fluency, IQ executive functioning, and attention, despite an increased risk to verbal memory associated with residual function in resected regions. ${ }^{7,8}$ The imaging literature also provides evidence for neuroplastic network changes with restorative potential. ${ }^{9,10}$ In a longitudinal fMRI study, plasticity of a working memory network was observed after temporal lobe surgery. ${ }^{9}$ Another study examining functional connectomes in the brainstem found that connectivity patterns of patients were more likely to resemble those of controls after epilepsy surgery. ${ }^{10}$ Other research, however, shows a limited effect of the procedure on functional and structural brain networks, ${ }^{32,33}$ and that connectivity normalization is associated with whether or not a patient becomes seizure-free as a result of neurosurgery. ${ }^{34,35}$

We expected that patients who were rendered completely seizure-free (ILAE 1) would have lower BrainAGE compared to patients with persistent postoperative symptoms (ILAE $2+)$. However, there was no significant difference between clinical outcome groups. Postoperative cognitive and qualityof-life outcomes are influenced by many factors and only partly depend on seizure control. ${ }^{36}$ BrainAGE may therefore reflect surgery-related changes that are not directly associated with seizure status. Furthermore, following the majority of previous epilepsy cohort studies, ${ }^{18}$ we used a dichotomous outcome grouping (ILAE 1 vs ILAE $2+$ ) that does not account for the considerable cumulative reduction in seizures for patients in the ILAE 2+ (PS) group. Notably, we did find a borderline difference between patients rendered seizure-free and only those who continued to experience debilitating postoperative seizures (ILAE 3-5), suggesting that there may in fact be a subtle relationship between BrainAGE and seizure outcome that may be difficult to detect in relation to surgery 
due to the personal improvements in seizure control that patients are likely to experience following surgery (an ILAE 3 patient who previously had a seizure a day will be a relative clinical success). Although we did not have access to patients' seizure outcome in response to antiseizure medication before surgery, it may be that BrainAGE reduction is more closely related to the difference between seizure outcome after compared to before surgery. Without information on postoperative antiseizure medication, it was also not possible to disentangle the effects of surgery from medication on seizure outcome. However, this does not have implications for our findings, as medication is not likely to have been significantly altered during the short period between pre- and postoperative scans (mean 1.96 years, SD 0.92).

Although we controlled for postoperative volume change and resection size in our statistical modeling, we cannot exclude the possibility that BrainAGE reduction may be a by-product of invasive surgery and unrelated to increased brain resilience. However, a supplementary analysis revealed that there was no significant BrainAGE difference between postoperative scans with artificially filled and unfilled resections, in accordance with a previous large-scale multiple sclerosis study, ${ }^{27}$ suggesting that the measure may capture features associated with brainwide correlates of surgery beyond those relating to presence of the surgical cavity. The link between postsurgery BrainAGE reduction and brain resilience could be more reliably established in future studies if a strong relationship between BrainAGE reduction and positive cognitive and quality of life outcomes is observed in a study with more postoperative scans to maximize the generalizability of study results. It may also be possible to isolate the BrainAGE correlates of surgery specifically related to TLE through comparison with a patient group without TLE that have undergone equivalent surgery treatment.

More generally, our results support the proposal that mTLE is related to morphologic changes of accelerated aging, in accordance with evidence associating the disorder with neurodegenerative features such as neuronal loss in and around the disease epicenter, ${ }^{37,38}$ axonal sprouting, ${ }^{39}$ blood-brain barrier leakage, ${ }^{40}$ loss of brain plasticity and reserve capacity, ${ }^{41}$ and increased inflammation. ${ }^{42}$ The extent of the BrainAGE increase ( 7.9 years) in patients before surgery found in our study is also consistent with previous epilepsy brain age studies that show comparable effects of between 4.5 and 10.9 years in focal epilepsy cohorts. ${ }^{15,16}$ These findings are in line with clinical and epidemiologic studies that highlight the benefits of earlier epilepsy surgery interventions over prolonged medical therapy. ${ }^{43-45}$

Our findings showed that patients with left mTLE were more likely to have normalized BrainAGE after surgery relative to patients with right mTLE, although no difference was found in resection size between left and right surgery. This is an additional novel finding and we can only speculate on the reasons why there is a lateralization difference. Surgery aside, it is well demonstrated that patients with left and right mTLE have different distributions of brain abnormalities: patients with left mTLE are frequently reported to have a more bilateral and widespread distribution of brain alterations relative to patients with right $\mathrm{mTLE}^{46}$ as well as a more intense progression of white and gray matter atrophy. ${ }^{47}$ A systematic review of neuropsychologic outcomes after epilepsy surgery showed that the greatest rate of improvement across all domains occurred in verbal fluency with left-sided temporal surgery. ${ }^{7}$ It has also been reported that surgery reduces mortality associated with refractory mTLE but only in patients with left-sided surgery. ${ }^{48}$ The same work indicated that mortality was not related to postoperative seizure outcome. In fact, there is little evidence to suggest that side of surgery is related to seizure outcome. Given the established link between BrainAGE and increased mortality, ${ }^{12}$ our findings, which should be taken as preliminary, leave open the possibility that surgery may have particular benefits for patients with left mTLE, although confirmation would require a targeted investigation that also measures surgery-related neuropsychologic changes.

BrainAGE algorithms are likely to improve in the future using larger training datasets and by taking advantage of multimodal imaging (e.g., T2- and diffusion-weighted MRI sequences). It is also not yet clear what 3D T1W image features most reliably contribute to the BrainAGE measure and further methodologic work in neurotypical and clinical populations is needed to better understand how it reflects the nonlinear patterns of agerelated changes including regional brain volume reductions. Given that there is a known pattern of cerebral (particularly limbic) atrophy in TLE, ${ }^{1,2}$ and that decreasing brain volume is a characteristic of aging, ${ }^{31}$ then increasing atrophy in TLE may drive greater BrainAGE. However, the factors that suggest a recovery of increasing BrainAGE after surgery is unknown.

Reliance on largely standardized MRI allows the method to be widely applicable in clinical settings where the sequence is acquired for all patients referred to epilepsy surgery programs. Based on our findings, brain-predicted age models have the potential to further risk stratify patients who will benefit from epilepsy surgery, thereby improving the personalized medicine approach for people with refractory epilepsy in conjunction with other imaging and neuropsychologic screening tools. ${ }^{14}$ Furthermore, given that increased BrainAGE is an independent predictor of mortality, ${ }^{12}$ the imaging biomarker may be used to identify patients at high risk of sudden unexplained death in epilepsy. Brain age models may play both a prognostic and diagnostic role in the neurocognitive and psychiatric disorders associated with epilepsy as these have proven to be useful in the context of psychiatric disease and impaired cognition. ${ }^{11,49}$ Other advances in deep learning applied to brain images are beginning to play a role in clinical decision-making, such as in the automatic classification and prognostics of TLE. ${ }^{50}$

Our study found that epilepsy surgery was associated with reduced brain imaging-defined age, suggesting that some 
morphologic brain changes linked with accelerated aging in mTLE may be reversible. This is consistent with studies that found neuropsychologic improvements following surgery and those calling for earlier surgical intervention where medical therapy is ineffective. Models of brain-predicted age may provide insight into the treatment and prognosis of epilepsy.

\section{Acknowledgment}

The authors thank Dr. James Cole for advice on applying the BrainAge R algorithm to postoperative scans in the supplementary lesion-filling analysis.

\section{Study Funding}

This work was supported by UK Medical Research Council grants (MR/S00355X/1 and MR/K023152/1) awarded to S.S. Keller.

\section{Disclosure}

The authors report no disclosures relevant to the manuscript. Go to Neurology.org/N for full disclosures.

\section{Publication History}

Received by Neurology June 11, 2020. Accepted in final form May 3, 2021.

Appendix Authors

\begin{tabular}{lll}
\hline Name & Location & Contribution \\
\hline $\begin{array}{l}\text { Christophe E. de } \\
\text { Bézenac, PhD }\end{array}$ & $\begin{array}{l}\text { University of } \\
\text { Liverpool, UK }\end{array}$ & $\begin{array}{l}\text { Design and conception of study, } \\
\text { analysis of data, drafting manuscript }\end{array}$ \\
\hline $\begin{array}{lll}\text { Guleed Adan, } \\
\text { MD }\end{array}$ & $\begin{array}{l}\text { University of } \\
\text { Liverpool, UK }\end{array}$ & Design and conception of study \\
\hline $\begin{array}{lll}\text { Bernd Weber, } \\
\text { MD, PhD }\end{array}$ & $\begin{array}{l}\text { University of } \\
\text { Bonn, Germany }\end{array}$ & Design and conception of study \\
\hline $\begin{array}{l}\text { Simon S. Keller, } \\
\text { PhD }\end{array}$ & $\begin{array}{l}\text { University of } \\
\text { Liverpool, UK }\end{array}$ & $\begin{array}{l}\text { Design and conception of study, } \\
\text { drafting manuscript }\end{array}$ \\
\hline
\end{tabular}

\section{References}

1. Janszky J, Janszky I, Schulz R, et al. Temporal lobe epilepsy with hippocampal sclerosis: predictors for long-term surgical outcome. Brain. 2005;128(pt 2):395-404

2. Farrell JS, Wolff MD, Teskey GC. Neurodegeneration and pathology in epilepsy: clinical and basic perspectives. Adv Neurobiol. 2017;15:317-334.

3. Berg AT. The natural history of mesial temporal lobe epilepsy. Curr Opin Neurol. 2008;21(2):173-178.

4. Engel J, McDermott MP, Wiebe S, et al. Early surgical therapy for drug-resistant temporal lobe epilepsy: a randomized trial. JAMA. 2012;307(9):922-930.

5. Spencer SS. When should temporal-lobe epilepsy be treated surgically? Lancet Neurol. 2002;1(6):375-382

6. Téllez-Zenteno JF, Dhar R, Wiebe S. Long-term seizure outcomes following epilepsy surgery: a systematic review and meta-analysis. Brain. 2005;128(pt 5):1188-1198.

7. Sherman EMS, Wiebe S, Fay-McClymont TB, et al. Neuropsychological outcomes after epilepsy surgery: systematic review and pooled estimates. Epilepsia. 2011;52(5): 857-869.

8. Helmstaedter C, Elger CE, Vogt VL. Cognitive outcomes more than 5 years after temporal lobe epilepsy surgery: remarkable functional recovery when seizures are controlled. Seizure. 2018;62:116-123.

9. Stretton J, Sidhu MK, Winston GP, et al. Working memory network plasticity after anterior temporal lobe resection: a longitudinal functional magnetic resonance imaging study. Brain. 2014;137(pt 5):1439-1453.

10. González HFJ, Goodale SE, Jacobs ML, et al. Brainstem functional connectivity disturbances in epilepsy may recover after successful surgery. Neurosurgery. 2020; $86(3): 417-428$
11. Gaser C, Franke K, Klöppel S, et al. BrainAGE in mild cognitive impaired patients: predicting the conversion to Alzheimer's disease. PLoS One. 2013;8(6):e67346.

12. Cole JH, Ritchie SJ, Bastin ME, et al. Brain age predicts mortality. Mol Psychiatry. 2018;23(5):1385-1392.

13. Smith SM, Vidaurre D, Alfaro-Almagro F, Nichols TE, Miller KL. Estimation of brain age delta from brain imaging. Neuroimage. 2019;200:528-539.

14. Franke K, Gaser C. Ten years of brain age as a neuroimaging biomarker of brain aging: what insights have we gained? Front Neurol. 2019;10:789.

15. Pardoe HR, Cole JH, Blackmon K, et al. Structural brain changes in medically refractory focal epilepsy resemble premature brain aging. Epilepsy Res. 2017;133:28-32.

16. Sone D, Beheshti I, Maikusa N, et al. Neuroimaging-based brain-age prediction in diverse forms of epilepsy: a signature of psychosis and beyond. Mol Psychiatry. 2019; 26(3):825-834.

17. Bien CG, Raabe AL, Schramm J, Becker A, Urbach H, Elger CE. Trends in presurgical evaluation and surgical treatment of epilepsy at one centre from 1988-2009. J Neurol Neurosurg Psychiatry. 2013;84(1):54-61.

18. Keller SS, Richardson MP, O’Muircheartaigh J, Schoene-Bake J-C, Elger C, Weber B. Morphometric MRI alterations and postoperative seizure control in refractory temporal lobe epilepsy. Hum Brain Mapp. 2015;36(5):1637-1647.

19. Kral T, Clusmann H, Urbach J, et al. Preoperative evaluation for epilepsy surgery (Bonn algorithm). Zentralblatt Neurochirurg. 2002;63(3):106-110.

20. Wieser H, Blume W, Fish D, et al. Commission on neurosurgery of the International League Against Epilepsy (ILAE). ILAE commission report: proposal for a new classification of outcome with respect to epileptic seizures following epilepsy surgery. Epilepsia. 2001;42(2):282-286

21. Ashburner J, Barnes G, Chen C, et al. SPM12 Manual. Wellcome Trust Centre for Neuroimaging; 2014:2464.

22. Karatzoglou A, Smola A, Hornik K, Zeileis A. Kernlab: an s4 package for kernel methods in R. J Stat Softw. 2004;11:1-20.

23. Le TT, Kuplicki RT, McKinney BA, et al. A nonlinear simulation framework supports adjusting for age when analyzing brain age. Front Aging Neurosci. 2018;10:317.

24. Smith SM, Zhang Y, Jenkinson M, et al. Accurate, robust, and automated longitudinal and cross-sectional brain change analysis. Neuroimage. 2002;17(1):479-489.

25. Zhang Y, Brady M, Smith S. Segmentation of brain MR images through a hidden Markov random field model and the expectation-maximization algorithm. IEEE Trans Med Imaging. 2001;20(1):45-57.

26. Battaglini M, Jenkinson M, De Stefano N. Evaluating and reducing the impact of white matter lesions on brain volume measurements. Hum Brain Mapp. 2012;33(9): 2062-2071.

27. Cole JH, Raffel J, Friede T, et al. Longitudinal assessment of multiple sclerosis with the brain-age paradigm. Ann Neurol. 2020;88(1):93-105.

28. Beheshti I, Nugent S, Potvin O, Duchesne S. Bias-adjustment in neuroimaging-based brain age frameworks: a robust scheme. Neuroimage Clin. 2019;24:102063.

29. Ho J, Tumkaya T, Aryal S, Choi H, Claridge-Chang A. Moving beyond p values: data analysis with estimation graphics. Nat Methods. 2019;16(7):565-566.

30. Lenth R. Estimated marginal means, aka least-squares means. R package version 1.3 . 5.1. 2019 .

31. Elliott ML, Belsky DW, Knodt AR, et al. Brain-age in midlife is associated with accelerated biological aging and cognitive decline in a longitudinal birth cohort. Mol Psychiatry. 2019;1-10.

32. Maccotta L, Lopez MA, Adeyemo B, et al. Postoperative seizure freedom does not normalize altered connectivity in temporal lobe epilepsy. Epilepsia. 2017;58(11): 1842-1851.

33. Taylor PN, Sinha N, Wang Y, et al. The impact of epilepsy surgery on the structural connectome and its relation to outcome. Neuroimage Clin. 2018;18:202-214.

34. Ji G-J, Zhang $\mathrm{Z}, \mathrm{Xu} \mathrm{Q}$, et al. Connectome reorganization associated with surgical outcome in temporal lobe epilepsy. Medicine. 2015;94(40):e1737.

35. Liao W, Ji G-J, Xu Q et al. Functional connectome before and following temporal lobectomy in mesial temporal lobe epilepsy. Sci Rep. 2016;6:23153.

36. Ryvlin P, Rheims S. Predicting epilepsy surgery outcome. Curr Opin Neurol. 2016; 29(2):182-188.

37. Bernhardt BC, Worsley K, Kim H, Evans A, Bernasconi A, Bernasconi N. Longitudinal and cross-sectional analysis of atrophy in pharmacoresistant temporal lobe epilepsy. Neurology. 2009;72(20):1747-1754.

38. Bernhardt BC, Kim H, Bernasconi N. Patterns of subregional mesiotemporal disease progression in temporal lobe epilepsy. Neurology. 2013;81(21):1840-1847.

39. Blümcke I, Thom M, Aronica E, et al. International consensus classification of hippocampal sclerosis in temporal lobe epilepsy: a task force report from the ILAE commission on diagnostic methods. Epilepsia. 2013;54(7):1315-1329.

40. Van Vliet E, da Costa Araujo S, Redeker S, Van Schaik R, Aronica E, Gorter J. Blood-brain barrier leakage may lead to progression of temporal lobe epilepsy. Brain. 2007; 130(pt 2):521-534

41. Breuer L, Boon P, Bergmans J, et al. Cognitive deterioration in adult epilepsy: does accelerated cognitive ageing exist? Neurosci Biobehav Rev. 2016;64:1-11.

42. Vezzani A, Friedman A. Brain inflammation as a biomarker in epilepsy. Biomarkers Med. 2011;5(5):607-614.

43. Bjellvi J, Olsson I, Malmgren K, Ramsay KW. Epilepsy duration and seizure outcome in epilepsy surgery: a systematic review and meta-analysis. Neurology. 2019;93(2): e159-e166.

44. Wiebe S, Blume WT, Girvin JP, Eliasziw M. A randomized, controlled trial of surgery for temporal-lobe epilepsy. N Engl J Med. 2001;345(5):311-318.

45. Wiebe S, Jette N. Pharmacoresistance and the role of surgery in difficult to treat epilepsy. Nat Rev Neurol. 2012;8(12):669. 
46. Bonilha L, Keller SS. Quantitative MRI in refractory temporal lobe epilepsy: relationship with surgical outcomes. Quant Imaging Med Surg. 2015;5(2):204.

47. Coan A, Appenzeller S, Bonilha L, Li L, Cendes F. Seizure frequency and lateralization affect progression of atrophy in temporal lobe epilepsy. Neurology. 2009;73(11):834-842. Hennessy M, Langan Y, Elwes R, Binnie C, Polkey C, Nashef L. A study of mortality after temporal lobe epilepsy surgery. Neurology. 1999;53(6):1276.
49. Koutsouleris N, Davatzikos C, Borgwardt S, et al. Accelerated brain aging in schizophrenia and beyond: a neuroanatomical marker of psychiatric disorders. Schizophr Bull. 2014;40(5):1140-1153.

50. Bernhardt BC, Hong S-J, Bernasconi A, Bernasconi N. Magnetic resonance imaging pattern learning in temporal lobe epilepsy: classification and prognostics. Ann Neurol. $2015 ; 77(3): 436-446$ 


\section{Neurology}

\section{Association of Epilepsy Surgery With Changes in Imaging-Defined Brain Age}

Christophe E. de Bézenac, Guleed Adan, Bernd Weber, et al.

Neurology 2021;97;e554-e563 Published Online before print July 14, 2021

DOI 10.1212/WNL.0000000000012289

This information is current as of July 14, 2021

\section{Updated Information \&} Services

References

Citations

Subspecialty Collections

Permissions \& Licensing

Reprints including high resolution figures, can be found at: http://n.neurology.org/content/97/6/e554.full

This article cites 47 articles, 6 of which you can access for free at: http://n.neurology.org/content/97/6/e554.full\#ref-list-1

This article has been cited by 1 HighWire-hosted articles: http://n.neurology.org/content/97/6/e554.full\#\#otherarticles

This article, along with others on similar topics, appears in the following collection(s):

Epilepsy surgery

http://n.neurology.org/cgi/collection/epilepsy_surgery_

Hippocampal sclerosis

http://n.neurology.org/cgi/collection/hippocampal_sclerosis

MRI

http://n.neurology.org/cgi/collection/mri

Information about reproducing this article in parts (figures,tables) or in its entirety can be found online at:

http://www.neurology.org/about/about_the_journal\#permissions

Information about ordering reprints can be found online:

http://n.neurology.org/subscribers/advertise

Neurology ${ }^{\circledR}$ is the official journal of the American Academy of Neurology. Published continuously since 1951, it is now a weekly with 48 issues per year. Copyright Copyright ( 2021 The Author(s). Published by Wolters Kluwer Health, Inc. on behalf of the American Academy of Neurology.. All rights reserved. Print ISSN: 0028-3878. Online ISSN: 1526-632X.

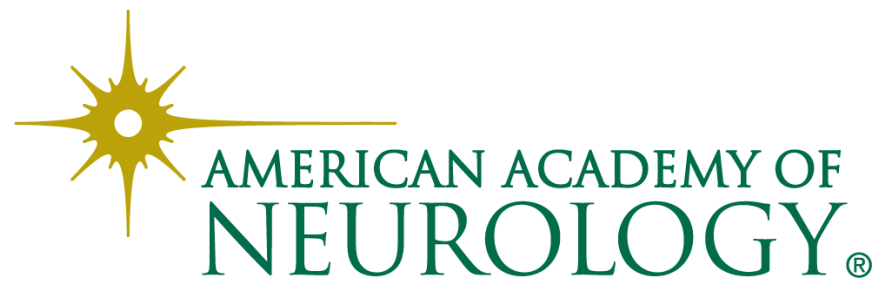

